\title{
existence,uniqueness solution for nonlinear mixed problem in two dimensional elasticity
}

\author{
Jaouad. Oudaani*, Enseignant au Lycée Qualifiant, Ajdir à Khénifra \\ 3 Rue Hôpital, Ain-Leuh, Province Ifran, Morocco \\ ${ }^{*}$ Corresponding author E-mail: oudaani1970@gmail.com
}

Copyright (C)2014 Jaouad. Oudaani et. al. This is an open access article distributed under the Creative Commons Attribution License, which permits unrestricted use, distribution, and reproduction in any medium, provided the original work is properly cited.

\begin{abstract}
The aim of this article is to minimises the stored energy function, of two dimensional elasticity with mixed boundary condition, in order, that the Euler's equilibrium equations of the Saint-Venant-Kirchhoff problem, has one and only one solution.
\end{abstract}

Keywords: Elasticity, Saint-Venant, Stored Energy.

\section{Introduction}

A special physical case, occurring an important place in nonlinear elasticity, is the Saint-Venant-Kirchhoff material whose the response function is given by the second Piola-Kirchhoff stress tensor,

$\Sigma(E)=\lambda(\operatorname{trace}(E)) I+2 \mu E$.

where $\lambda$ and $\mu$ are two constants, known as the Lamé coefficients, and

$E:=E(\nabla u)=1 / 2\left((\nabla u)^{t}(\nabla u)+(\nabla u)^{t}+(\nabla u)\right)$

is the nonlinear Green-Saint-Venant strain tensor, $\nabla u$ is the displacement gradient.

The mathematical problem consists in solving a nonlinear boundary value problem, with mixed Dirichlet and Neuman conditions, for the displacement $u$ such that

(S)

$$
\left\{\begin{aligned}
-\operatorname{div}((I+\nabla u) \Sigma(E(\nabla u))) & =f \quad \text { in } \Omega \\
(I+\nabla u) \Sigma(E(\nabla u)) \cdot \vec{n} & =g \text { on } \Gamma_{1}, \\
u & =0 \text { on } \Gamma_{0} .
\end{aligned}\right.
$$

The linearized mixed problem of $(S)$ is

$\left(S_{l}\right)\left\{\begin{aligned}-\operatorname{div}(\Sigma(\epsilon(\nabla u))) & =f \quad \text { in } \Omega, \\ \Sigma(\epsilon(\nabla u)) \cdot \vec{n} & =g \quad \text { on } \Gamma_{1}, \\ u & =0 \quad \text { on } \Gamma_{0} .\end{aligned}\right.$

where $\epsilon(u)=1 / 2\left((\nabla u)^{t}+(\nabla u)\right)$ is the linear Green-Saint-Venant strain.

In [1], J.M.Ball introduces the notion of polyconvexity and minimizes the stored energy to study the existence of solutions to the mixed boundary value problem of nonlinear elasticity for a wide class of hyperelastic materials, which does not include the Saint-Venant-Kirchhoff material, because its stored energy function, as shown by A.Raoult, is not polyconvex(then neither convex). The assumptions $\lambda>0$ and $\mu>0$ satisfied by the Lamé coefficients are two physical conditions as shown by an experimental evidence. But many authors(see[3] for example) included the case $\lambda=0$ corresponding to "limit" of Saint-Venant-Kirchhoff material in the sense that $\lambda$ is physically very small 
$(\lambda \approx 0)$. Mathematically, this " limit " case plays an important part for the two next reasons: Firstly, the Ciarlet's existence theory for the pure displacement problem(i.e. $\Gamma_{1}=\emptyset$ ) still hold under the weaker assumptions $\mu>0$ and $\lambda+2 \mu>0$, see [2, chapter 6$]$ and [5, Section6.1]. The second reason is explained by the fact that the study of the nonlinear problem $(S)$ for $\lambda=0$ is more difficult than other cases, since M.Atteia and M.Raissouli showed, see $[2,7]$, that the associated stored energy function for $\lambda=0$ defined by: $J(u)=1 / 4 \int_{\Omega}|E(\nabla u)|^{2}-\int_{\Omega} f u-\int_{\Gamma_{1}} g u$ can't be convex. For the previous arguments, we limit our attention throughout the following, not to lengthen the paper, to the case $\lambda=0$ and $\mu=1 / 2$ that corresponds to $\Sigma(E)=E$.

The fundamental goal of this work is to prove, existence and uniqueness solution to the problem of minimizing the stored energy, in two dimensional case.

The paper is organized as follows. In section 1 describes the formulation of the problem which we will study later. Section 2 is devoted to introduce some preliminary results. In the final Section, we state our fundamental theorem concerning the local existence, uniqueness of solution for the nonlinear mixed problem.

\section{Formulation of the problem}

In this paper, $\Omega$ denotes a nonempty bounded open domain in $I R^{2}$, with its boundary $\Gamma=\partial \Omega$ of $C^{\infty}$-regularity. We assume that $\Gamma=\Gamma_{o} \cup \Gamma_{1}$ where $\Gamma_{0}$ and $\Gamma_{1}$ are two measurable portions of $\Gamma$ with $\Gamma_{o} \cup \Gamma_{1}=\emptyset$.

Let $f \in\left(L^{2}(\Omega)\right)^{2}$ and $g \in\left(H^{1 / 2}\left(\Gamma_{1}\right)\right)^{2}$. Let us consider the following problem:

Fund $u \in\left(W^{1,4}(\Omega)\right)^{2}$ such that

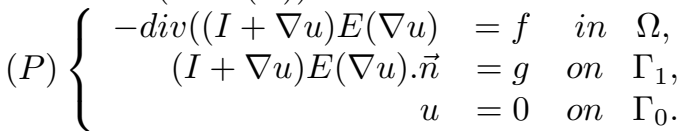

Where $\mathrm{I}$ is the identity matrix, $\vec{n}$ is the exterior normal vector to $\Gamma$ and

$E(\nabla u)=1 / 2\left(\nabla u^{t} \nabla u+\nabla u^{t}+\nabla u\right)$,

is the nonlinear Green-Saint-Venant strain tensor.

The linearized problem of $(P)$ is the following:

Fund $u \in\left(H^{1}(\Omega)\right)^{2}$ such that

$\left(P_{l}\right)\left\{\begin{aligned}-\operatorname{div}(\epsilon(u)) & =f \quad \text { in } \quad \Omega, \\ \epsilon(u) \cdot \vec{n} & =g \text { on } \Gamma_{1}, \\ u & =0 \text { on } \Gamma_{0} .\end{aligned}\right.$

Where $\epsilon(u)=\left(\epsilon_{i, j}(u)\right)_{1 \leq i, j \leq 2}=1 / 2\left(\nabla u^{t}+\nabla u\right)$,

is the linearized strain tensor.

Let $\mathrm{u}$ and $\mathrm{v}$ in $\left(W^{1,4}(\Omega)\right)^{2}$, we defined by, [7]

$<\nabla u / \nabla v>=\sum_{i, j=1}^{2} \partial_{j} u_{i} \partial_{j} v_{i}$

is an inner scalar product with its associated norm:

$|\nabla u|=<\nabla u / \nabla v>^{1 / 2}=\left(\sum_{i, j=1}^{2}\left(\partial_{j} u_{i}\right)^{2}\right)^{1 / 2}$

We shall study the problem $(P)$ : Existence and uniqueness solution, by considering the following problem [7]

$(Q) J(u)=\inf \left\{J(v), v \in\left(W^{1,4}(\Omega)\right)^{2}, v=0\right.$ on $\left.\Gamma_{0}, \operatorname{det}(I+\nabla v)>0\right\}$

Where $J(u)=1 / 4 \int_{\Omega}|E(\nabla u)|^{2}-\int_{\Omega} f u-\int_{\Gamma_{1}} g u$

is the stored energy function of mixed elasticity problem [7].

\section{Existence and uniqueness solution of problem $(P)$}

In this short section, we recall some standard notation and results. For some details, one can consult [4]

$V=\left\{u \in\left(W^{1,4}(\Omega)\right)^{2}, u=0\right.$ on $\left.\Gamma_{0}\right\}$

clearly $\mathrm{V}$ is a closed vector subspace of $\left(W^{1,4}(\Omega)\right)^{2}$.

For every $u \in\left(W^{1,4}(\Omega)\right)^{2}$ we define

$|\epsilon(u)|_{0 . \Omega}^{2}=\sum_{i, j=1}^{2} \int_{\Omega}\left(\epsilon(u)_{i j}\right)^{2}$.

Proposition 3.1 The semi-norm $|\epsilon(u)|_{0 . \Omega}$ is a norm in $V \cap\left(H^{1}(\Omega)\right)^{2}$ equivalent to the norm usual, $\|\cdot\|$ of $\left(H^{1}(\Omega)\right)^{2}$. Proof. Since $|\epsilon(u)|_{0 . \Omega}$ is a norm in $V \cap\left(H^{1}(\Omega)\right)^{2}$ and equivalent to the norm of $\left(H^{1}(\Omega)\right)^{2}[4]$, is a norm in $V \cap\left(H^{1}(\Omega)\right)^{2}$.

Lemma 3.2 [2] Let $F$ be a normed vector space, $G$ is a Banach space and: $B \times G \longmapsto I R$ be a continuous bilinear maping. Then for all sequences $v_{n}$ strongly converges to $v$ in $F$ and $w_{n}$ weakly converges to $w$ in $G$ we have, $B\left(v_{n}, w_{n}\right)$ converge to $B(v, w)$. 
Theorem 3.3 [4] Let $F$ be a reflexif, Banach space and $j: F \longmapsto I R$ is a lower semi-continuous, convex, coercive functional, then the problem $j(u)=\inf \{j(v) ; v \in F\}$ has one and only one solution.

Theorem 3.4 [7] Let $f \in\left(L^{2}(\Omega)\right)^{2}$ and $g \in\left(H^{1 / 2}\left(\Gamma_{1}\right)\right)^{2}$ then, $u$ is a uniqueness solution of $(P)$ if and only if $u$ is also a uniqueness solution of $(Q)$ in $V$.

Lemma $3.5[6]$ the following map:

$p\left(W^{1,4}(\Omega)\right)^{2} \longmapsto I R$

$$
u \quad 1 \quad 1 / 16\left(\int_{\Omega}\left|\nabla u^{t} \nabla u\right|^{2}\right)^{1 / 4}
$$

is a lower semi-continuous, convex and satisfying the triangular inequality(see[6, chapter 2 , page69]).

Lemma 3.6 Let $W$ be a neighborhood of 0 in $\left(W^{1,4}(\Omega)\right)^{2}$, then there exists $0<C_{1}<1$ such that for evry $u \in W$ and $v \in W$ we have: $\|F(u)-F(v)\|_{1} \leq C_{1}\|u-v\|_{\left(H^{1}(\Omega)\right)^{2}}$,

where $F(u)=\nabla u E(\nabla u)+1 / 2\left((\nabla u)^{t} \nabla u\right)$.

Proof. Let $\mathrm{u}, \mathrm{v}$ in $\left(W^{1,4}(\Omega)\right)^{2}$ such that $\|u\|_{\left(W^{1,4}(\Omega)\right)^{2}} \leq C,\|v\|_{\left(W^{1,4}(\Omega)\right)^{2}} \leq C$ where $\quad C>0$ is small. We have $\|F(u)-F(v)\|_{1}=\sum_{i=1}^{2}\left\|\sum_{j=1}^{2}\left(F_{i j}(u)-F_{i j}(v)\right)\right\|_{1} \leq \sum_{i, j=1}^{2}\left\|F_{i j}(u)-F_{i j}(v)\right\|_{1}$

and $F_{i j}=(1 / 2) \sum_{k=1}^{2} \partial_{i} u_{k} \partial_{j} u_{k}+(1 / 2) \sum_{k, r=1}^{2} \partial_{k} u_{i} \partial_{r} u_{k} \partial_{r} u_{j}+(1 / 2) \sum_{k=1}^{2} \partial_{k} u_{i} \partial_{k} u_{j}+(1 / 2) \sum_{k=1}^{2} \partial_{k} u_{i} \partial_{j} u_{k}$

For $\mathrm{i}, \mathrm{j}=1,2$

$\left\|\partial_{i} u_{k} \partial_{j} u_{k}-\partial_{i} v_{k} \partial_{j} v_{k}\right\|_{1} \leq\left\|\partial_{i} u_{k}\left(\partial_{j} u_{k}-\partial_{j} v_{k}\right)\right\|_{1}+\left\|\partial_{j} v_{k}\left(\partial_{i} u_{k}-\partial_{i} v_{k}\right)\right\|_{1}$

Since for $i, j=1,2, \partial_{j} u_{i} \in\left(L^{4}(\Omega)\right)^{2}$, we deduce that:

$\left.\left.\left\|\partial_{i} u_{k} \partial_{j} u_{k}-\partial_{i} v_{k} \partial_{j} v_{k}\right\|_{1} \leq\left\|\partial_{i} u_{k}\right\|_{2} \| \partial_{j} u_{k}-\partial_{j} v_{k}\right)\left\|_{2}+\right\| \partial_{j} v_{k}\left\|_{2}\right\| \partial_{i} u_{k}-\partial_{i} v_{k}\right) \|_{2}$

It becomes that:

$\left\|\partial_{i} u_{k} \partial_{j} u_{k}-\partial_{i} v_{k} \partial_{j} v_{k}\right\|_{1} \leq 2 K C\|u-v\|_{\left(H^{1}(\Omega)\right)^{2}}$,

where $K>0$ is a constant.

By an analogous method (see[9]), we prove that for $\mathrm{i}, \mathrm{j}, \mathrm{k}, \mathrm{r}=1,2$

$\left\|\partial_{i} u_{k} \partial_{j} u_{k} \partial_{r} u_{k}-\partial_{i} v_{k} \partial_{j} v_{k} \partial_{r} v_{k}\right\|_{1} \leq 3 K C^{2}\|u-v\|_{\left(H^{1}(\Omega)\right)^{2}}$,

Then for $\mathrm{i}, \mathrm{j}=1,2$

$\left\|F_{i j}(u)-F_{i j}(v)\right\|_{1} \leq 6 K C\|u-v\|_{\left(H^{1}(\Omega)\right)^{2}}+12 K C^{2}\|u-v\|_{\left(H^{1}(\Omega)\right)^{2}}$,

In summary, we have proved

$\|F(u)-F(v)\|_{1} \leq \max \left\{24 K C, 46 K C^{2}\right\}\|u-v\|_{\left(H^{1}(\Omega)\right)^{2}}$,

Put that $C_{1}=\max \left\{24 K C, 48 K C^{2}\right\}$

If $\mathrm{C}$ is sufficiently small then $0<C_{1}<1$ and

$\|F(u)-F(v)\|_{1} \leq C_{1}\|u-v\|_{\left(H^{1}(\Omega)\right)^{2}}$.

\section{Remark 3.7.}

1. In the space $V$, the norm:

$\|u\|_{1, \Omega}=\left(\int_{\Omega}|\epsilon(u)|^{2}\right)^{1 / 2}+1 / 16\left(\int_{\Omega}\left|(\nabla u)^{t} \nabla u\right|^{2}\right)^{1 / 4}$,

is equivalent to the classical norm of $\left(W^{1,4}(\Omega)\right)^{2}$.

2. For all $u \in V$ we have

$|E(\nabla u)|^{2}=|\epsilon(u)|^{2}+1 / 4\left|(\nabla u)^{t} \nabla u\right|^{2}+<\epsilon(u) /(\nabla u)^{t} \nabla u>$.

New we shall study the problem $(Q)$ by considering the following iterative problem:

Let $n \geq 0,0 \neq u_{0} \in\left(W^{1,4}(\Omega)\right)^{2}$ is given, $u_{n} \in\left(W^{1,4}(\Omega)\right)^{2}$ is constricted, when $u_{n-1}$ is known, as a solution of the following problem $\left(Q_{n}\right)$ :

$\left(Q_{n}\right) \quad J_{n}\left(u_{n}\right)=\inf \left\{J_{n-1}(u) ; u \in\left(W^{1,4}(\Omega)\right)^{2}\right\}$,

where $J_{n}(u)=\int_{\Omega}|\epsilon(u)|^{2}+1 / 16\left(\int_{\Omega}\left|(\nabla u)^{t} \nabla u\right|^{2}\right)+1 / 2 \int_{\Omega}<\epsilon(u) /\left(\nabla u_{n-1}\right)^{t} \nabla u_{n-1}>-\int_{\Omega} f u-\int_{\Gamma_{1}} g u$

Theorem 3.8 Let $f \in\left(L^{2}(\Omega)\right)^{2}$ and $g \in\left(H^{1 / 2}\left(\Gamma_{1}\right)\right)^{2}$, then the functional:

$J_{n}\left(W^{1,4}(\Omega)\right)^{2} \longmapsto I R$ is lower semi-continuous, convex and coercive.

Proof. Since the following maps:

$u \longmapsto \int_{\Omega}|\epsilon(u)|^{2}+1 / 16\left(\int_{\Omega}\left|(\nabla u)^{t} \nabla u\right|^{2}\right)$, and $u \longmapsto 1 / 2 \int_{\Omega}<\epsilon(u) /\left(\nabla u_{n-1}\right)^{t} \nabla u_{n-1}>-\int_{\Omega} f u-\int_{\Gamma_{1}} g u$,

are semi-continuous and convex, then $J_{n}$ is semi-continuous and convex.

Let $u \in\left(W^{1,4}(\Omega)\right)^{2}$ we have:

$\left|\int_{\Omega}<\epsilon(u) /\left(\nabla u_{n-1}\right)^{t} \nabla u_{n-1}>\right| \leq K_{1}\|\epsilon(u)\|_{2}\left\|\nabla u_{n-1}\right\|_{4}^{2}$,

$\left|\int_{\Omega} f u+\int_{\Gamma_{1}} g u\right| \leq K_{2}\left(\|f\|_{2}+\|g\|_{\left(H^{1 / 2}\left(\Gamma_{1}\right)\right)^{2}}\right)\|\epsilon(u)\|_{2}$, hence $J_{n}(u) \geq\|\epsilon(u)\|_{2}^{2}-K_{1}\|\epsilon(u)\|_{2}\left\|\nabla u_{n-1}\right\|_{4}^{2}-K_{2}\left(\|f\|_{2}+\right.$ 
$\left.\|g\|_{\left(H^{1 / 2}\left(\Gamma_{1}\right)\right)^{2}}\right)\|\epsilon(u)\|_{2}+1 / 16\left(\int_{\Omega}\left|(\nabla u)^{t} \nabla u\right|^{2}\right)$.

If $\|u\| \longmapsto+\infty$, the quantity

$\|\epsilon(u)\|_{2}\left(\|\epsilon(u)\|_{2}-K_{1}\left\|\nabla u_{n-1}\right\|_{4}^{2}-K_{2}\left(\|f\|_{2}+\|g\|_{\left(H^{1 / 2}\left(\Gamma_{1}\right)\right)^{2}}\right)\right.$ converge to $+\infty$,

also $J_{n}(u) \longmapsto+\infty$, when $\|u\| \longmapsto+\infty$, lastly the functional $J_{n}(u)$ is coercive.

Theorem 3.9 Let $f \in\left(L^{2}(\Omega)\right)^{2}$ and $g \in\left(H^{1 / 2}\left(\Gamma_{1}\right)\right)^{2}$, the problem $\left(Q_{n}\right)$ has one and only one solution $u_{n}$ in $V$.

Proof. Since the space V is closed in the reflexif space $\left(W^{1,4}(\Omega)\right)^{2}$ and $J_{n}(u)$ is lower semi-continuous, convex and coercive, afterward Theorem 2.1. the problem $\left(Q_{n}\right)$ has one and only one solution $u_{n}$ in V.

Theorem 3.10 Let $f \in\left(L^{2}(\Omega)\right)^{2}$ and $g \in\left(H^{1 / 2}\left(\Gamma_{1}\right)\right)^{2}$, the problem $(Q)$ has one and only one solution in $V$.

Poof. We divide this proof in three steps:

First step:. We will prove that for $C>0$

$\forall n \in I N, \int_{\Omega}\left|\epsilon\left(u_{n}\right)\right|^{2} \leq C$ and $1 / 16\left(\int_{\Omega}\left|\left(\nabla u_{n}\right)^{t} \nabla u_{n}\right|^{2}\right)^{1 / 2} \leq C$,

We assume that for all

$k=0,1, \ldots, n-1, C>0, \int_{\Omega}\left|\epsilon\left(u_{k}\right)\right|^{2} \leq C, 1 / 16\left(\int_{\Omega}\left|\left(\nabla u_{k}\right)^{t} \nabla u_{k}\right|^{2}\right)^{1 / 2} \leq C$,

$u_{n}$ is a solution of the problem $\left(Q_{n}\right)$ then $J_{n}\left(u_{n}\right) \leq J_{n}(0)=0$

hence $\left\|\epsilon\left(u_{n}\right)\right\|_{2}^{2} \leq 1 / 2 \int_{\Omega}<\epsilon\left(u_{n}\right) /\left(\nabla u_{n-1}\right)^{t} \nabla u_{n-1}>+\int_{\Omega} f u_{n}+\int_{\Gamma_{1}} g u_{n}$

then $\left\|\epsilon\left(u_{n}\right)\right\|_{2}^{2} \leq 1 / 2\left\|\epsilon\left(u_{n}\right)\right\|_{2}\left\|\left(\nabla u_{n-1}\right)^{t} \nabla u_{n-1}\right\|_{2}+K(f, g)\left\|\epsilon\left(u_{n}\right)\right\|_{2}$

where $K(f, g)=K_{2}\left(\|f\|_{2}+\|g\|_{H^{1 / 2}\left(\Gamma_{1}\right)^{2}}\right)$

Since $\left\|\left(\nabla u_{n-1}\right)^{t} \nabla u_{n-1}\right\|_{2} \leq 16 C$

then $\left\|\epsilon\left(u_{n}\right)\right\|_{2} \leq 8 C+K(f, g)$

if we choose $0<C<1 / 64$ and $K(f, g) \leq\left(1-8 C^{1 / 2}\right) C^{1 / 2}$ we obtain that

$\left\|\epsilon\left(u_{n}\right)\right\|_{2}^{2} \leq \int_{\Omega}|\epsilon(u)|^{2} \leq C$

by virtue of the inequality $J_{n}\left(u_{n}\right) \leq 0$, we have

$\left\|\epsilon\left(u_{n}\right)\right\|_{2}^{2}+1 / 16\left(\int_{\Omega}\left|\left(\nabla u_{n}\right)^{t} \nabla u_{n}\right|^{2}\right) \leq 1 / 2\left\|\epsilon\left(u_{n}\right)\right\|_{2}\left\|\left(\nabla u_{n-1}\right)^{t} \nabla u_{n-1}\right\|_{2}+K(f, g)\left\|\epsilon\left(u_{n}\right)\right\|_{2}$

and $\left\|\left(\nabla u_{n}\right)^{t} \nabla u_{n}\right\|_{2}^{2} \leq 128 C^{3 / 2}+16 K(f, g) C^{1 / 2}-16 C=16 C^{1 / 2}\left(8 C+K(f, g)-C^{1 / 2}\right)$

if we choose $\mathrm{K}(\mathrm{f}, \mathrm{g})$ satisfying $8 C+K(f, g)-C^{1 / 2} \leq 16 C^{3 / 2}$

is equivalent to have $C>0$ and $K(f, g) \leq C^{1 / 2}\left(4 C^{1 / 2}-1\right)^{2}$

we have $1 / 16\left(\int_{\Omega}\left|\left(\nabla u_{n}\right)^{t} \nabla u_{n}\right|^{2}\right)^{1 / 2} \leq C$

then for all $n \in I N,\left\|u_{n}\right\|_{1, \Omega} \leq C^{1 / 2}+(16 C)^{4} / 16$

and the sequence $\left(u_{n}\right)_{n \geq 0}$ is borned in the reflexif space $\left(W^{1,4}(\Omega)\right)^{2}$, hence we can extract a subsequence $\left(u_{m}\right)_{m \geq 0}$ weakly convergent to $\mathrm{u}$ in $\mathrm{V}$.

Second step: We show that $J_{m}\left(u_{m}\right)$ converge to $J(u)$ or

$J_{m}(u)=\int_{\Omega}|\epsilon(u)|^{2}+1 / 16\left(\int_{\Omega}\left|(\nabla u)^{t} \nabla u\right|^{2}\right)+1 / 2 \int_{\Omega}<\epsilon(u) /\left(\nabla u_{m-1}\right)^{t} \nabla u_{m-1}>-\int_{\Omega} f u_{m}-\int_{\Gamma_{1}} g u_{m}$

Since the functional

$u \longmapsto \int_{\Omega}|\epsilon(u)|^{2}+1 / 16\left(\int_{\Omega}\left|(\nabla u)^{t} \nabla u\right|^{2}\right)-\int_{\Omega} f u-\int_{\Gamma_{1}} g u$

is lower semi-continuous and convex, then the quantity

$\int_{\Omega}\left|\epsilon\left(u_{m}\right)\right|^{2}+1 / 16\left(\int_{\Omega}\left|\left(\nabla u_{m}\right)^{t} \nabla u_{m}\right|^{2}\right)-\int_{\Omega} f u_{m}-\int_{\Gamma_{1}} g u_{m}$ converge to $\int_{\Omega}|\epsilon(u)|^{2}+1 / 16\left(\int_{\Omega}\left|(\nabla u)^{t} \nabla u\right|^{2}\right)-\int_{\Omega} f u-\int_{\Gamma_{1}} g u$ If we have $\int_{\Omega}<\epsilon\left(u_{m}\right) /\left(\nabla u_{m}\right)^{t} \nabla u_{m-1}>$ converge to $\int_{\Omega}<\epsilon(u) /(\nabla u)^{t} \nabla u>$

then $J_{m}\left(u_{m}\right)$ converge to $J(u)$, or

$2 \int_{\Omega}<\epsilon\left(u_{m}\right) / \nabla u_{m}^{t} \nabla u_{m-1}>-<\epsilon(u) / \nabla u^{t} \nabla u>=\int_{\Omega} \underbrace{<\nabla u_{m} / \nabla u_{m-1}^{t} \nabla u_{m-1}>-<\nabla u / \nabla u^{t} \nabla u>}_{(1)}$.

$$
+\int_{\Omega} \underbrace{<\nabla u_{m}^{t} / \nabla u_{m-1}^{t} \nabla u_{m-1}>-<\nabla u^{t} / \nabla u^{t} \nabla u>}_{(2)}
$$

if we show that (1) converge to zero then, (2) is also convergent to zero because the proof is similarly to the (1)

$(1)=\int_{\Omega} \underbrace{<\left(\nabla u_{m}\right)^{t}-\nabla u /\left(\nabla u_{m-1}\right)^{t} \nabla u_{m-1}>}_{A}+\int_{\Omega} \underbrace{<(\nabla u)^{t} /\left(\nabla u_{m-1}\right)^{t} \nabla u_{m-1}-(\nabla u)^{t} \nabla u>}_{B}$

Since the map:

$u \longmapsto 1 / 16\left(\int_{\Omega}\left|(\nabla u)^{t} \nabla u\right|^{2}\right)$ is semi-continuous, convex and the subsequence $\left(u_{m}\right)_{m \geq 0}$ converge weakly to $\mathrm{u}$ in $\left(W^{1,4}(\Omega)\right)^{2}$.

Then $\int_{\Omega}\left|\left(\nabla u_{m-1}\right)^{t} \nabla u_{m-1}\right|^{2}$ converge to $\int_{\Omega}\left|(\nabla u)^{t} \nabla u\right|^{2}$,

hence the sequence $\left|\left(\nabla u_{m-1}\right)^{t} \nabla u_{m-1}\right|$ is strongly convergent to $\left|\nabla u^{t} \nabla u\right|$ in $\left(L^{2}(\Omega)\right)^{4}$ and the sequence $\nabla u_{m}$ converge weakly to $\nabla u$ afterware the Lemma 2.1 we have A converge to zero. 
we have $B=\int_{\Omega}<\nabla u^{t} / \nabla u_{m-1}^{t} \nabla u_{m-1}-\nabla u^{t} \nabla u>\leq\left\|\nabla u^{t}\right\|_{2}\left\|\nabla u_{m-1}^{t} \nabla u_{m-1}-\nabla u^{t} \nabla u\right\|_{2}$ then, B converge to zero. finally, $J_{m}\left(u_{m}\right)$ converge to $J(u)$ and for all $v \in V, J_{m}\left(u_{m}\right) \leq J_{m}(v)$

Since, $J_{m}\left(u_{m}\right)$ converge to $J(u)$ and $J_{m}(v)$ converge to $J(v)$

then, for all $\mathrm{v}$ in $\mathrm{V} \quad J(u) \leq J(v)$

hence, $\mathrm{u}$ is a solution of the problem $(Q)$.

Third step: Uniqueness of the problem $(Q)$.

Let $u_{1}$ and $u_{2}$ are two solutions of $(Q)$, then $u_{1}$ and $u_{2}$ are also solutions of $(P)$.

we have $-\operatorname{div}((I+\nabla u) E(\nabla u)=-\operatorname{div}(\epsilon(u))-\operatorname{div}(F(u))$,

where $F(u)=\nabla u E(\nabla u)+1 / 2 \nabla u^{t} \nabla u$.

We have

$\left\{\begin{aligned}-\operatorname{div}\left(\epsilon\left(u_{1}-u_{2}\right)\right) & =-\operatorname{div}\left(F\left(u_{2}\right)-F\left(u_{1}\right)\right) & & \text { in } \Omega, \\ \epsilon\left(u_{1}-u_{2}\right) \cdot \vec{n} & =\left(F\left(u_{2}\right)-F\left(u_{1}\right)\right) \cdot \vec{n} & & \text { on } \Gamma_{1}, \\ u_{1}-u_{2} & =0 & & \text { on } \Gamma_{0} .\end{aligned}\right.$

We put by $f_{1}=-\left(\operatorname{div}\left(F\left(u_{2}\right)-F\left(u_{1}\right)\right)\right.$ and $g_{1}=\left(F\left(u_{2}\right)-F\left(u_{1}\right)\right) \cdot \vec{n}$

we consider the linear operator

div : $\left.L^{1}(\Omega)\right)^{2} \longrightarrow\left(W^{-2,4}(\Omega)\right)^{2}$

$u \longmapsto \operatorname{div}(u)$

is continuous, And

trace $:\left(W^{-2,4}(\Omega)\right)^{2} \longrightarrow\left(W^{-2 / 3,4}\left(\Gamma_{1}\right)\right)^{2}$

$$
u \quad \operatorname{trace}(u)=u \cdot \vec{n}
$$

is linear and continuous, Because, let $\left.u \in L^{1}(\Omega)\right)^{2}$ and for all $v \in\left(W_{0}^{2,4}(\Omega)\right)^{2}$ we have $\int_{\Omega}-\operatorname{div}(u) v=\int_{\Omega} u \operatorname{div}(v) \leq\|u\|_{1} \underbrace{\sup }_{\bar{\Omega}}|\operatorname{div}(u)|$

since $\left(W_{0}^{2,4}(\Omega)\right)^{2} \hookrightarrow\left(C^{1}(\bar{\Omega})\right)^{2}$, then there exist a $K^{\prime}>0$ such that $\underbrace{\sup }_{\bar{\Omega},|\alpha| \leq 1}\left|D^{\alpha} u\right| \leq K^{\prime}\|u\|_{\left(W_{0}^{2,4}(\Omega)\right)^{2}}$

and also we have, for all $\mathrm{v}$ in $\left(W_{0}^{2,4}(\Omega)\right)^{2} \int_{\Omega} \operatorname{div}(u) v \leq K^{\prime}\|u\|_{1}\|v\|_{\left(W_{0}^{2,4}(\Omega)\right)^{2}}$

then $\|\operatorname{div}(u)\|_{\left(W^{-2,4}(\Omega)\right)^{2}}=\underbrace{\sup }_{v \in\left(W_{0}^{2,4}(\Omega)\right)^{2}} \frac{\int_{\Omega} \operatorname{div(u)v}}{\|v\|} \leq K^{\prime}\|u\|_{1}$

We conclude that the operator div is continuous, for the operator trace there is also continuous.

by similarly next proof we can show that $\left.L^{1}(\Omega)\right)^{2} \hookrightarrow\left(W^{-2,4}(\Omega)\right)^{2}$

Now we put by:

$$
\begin{aligned}
A: V & \longrightarrow\left(W^{-2,4}(\Omega)\right)^{2} \times\left(W^{-3 / 2,4}\left(\Gamma_{1}\right)\right)^{2} \\
u & \longmapsto A(u)=(-\operatorname{div}((I+\nabla u) E(\nabla u)),(I+\nabla u) E(\nabla u) \cdot \vec{n})
\end{aligned}
$$

$A$ is, defined, infinitely Frechet differentiable and $A(0)=0$ and the linear operator:

$$
\begin{aligned}
A^{\prime}(O): V & \longrightarrow\left(W^{-2,4}(\Omega)\right)^{2} \times\left(W^{-3 / 2,4}\left(\Gamma_{1}\right)\right)^{2} \\
u & \longmapsto A^{\prime}(0)(u)=(-\operatorname{div}(\epsilon(u)), \epsilon(u) \cdot \vec{n})
\end{aligned}
$$

is infinitely Frechet differentiable.

afterware, $[4,8]$ the problem $A^{\prime}(O)=\left(f_{1}, g_{1}\right)$ has one and only one solution $\mathrm{u}$ in $\left(H^{1}(\Omega)\right)^{2}$, since $u_{1}-u_{2}$ is a solution of the problem $A^{\prime}(O)=\left(f_{1}, g_{1}\right)$ in $\mathrm{V}$, then $u=\left(u_{1}-u_{2}\right) \in V$ and satisfy that $\left\|u_{1}-u_{2}\right\|_{\left.H^{1}(\Omega)\right)^{2}} \leq K\left(\| \operatorname{div}\left(F\left(u_{2}\right)-F\left(u_{1}\right)\left\|_{\left(W^{-2,4}(\Omega)\right)^{2}}+\right\| F\left(u_{2}\right)-F\left(u_{1}\right) \|_{\left(W^{-3 / 2,4}\left(\Gamma_{1}\right)\right)^{2}}\right)\right.$ afterware, $\left(L^{1}(\Omega)\right)^{2} \hookrightarrow\left(W^{-2,4}(\Omega)\right)^{2}$, and the continuous of the operator trace there exist $K_{1}>0$ such that $\left\|u_{1}-u_{2}\right\|_{\left.H^{1}(\Omega)\right)^{2}} \leq K_{1}\left(\left\|F\left(u_{2}\right)-F\left(u_{1}\right)\right\|_{1}+\left\|F\left(u_{2}\right)-F\left(u_{1}\right)\right\|_{\left(W^{-2,4}(\Omega)^{2}\right.}\right)$

and we have $\left\|u_{1}-u_{2}\right\|_{\left.H^{1}(\Omega)\right)^{2}} \leq K_{2}\left\|F\left(u_{2}\right)-F\left(u_{1}\right)\right\|_{1}$ afterware the Lemma 2.3. If we have $\left\|u_{1}\right\|_{\left.H^{1}(\Omega)\right)^{2}} \leq C$ and $\left\|u_{2}\right\|_{\left.H^{1}(\Omega)\right)^{2}} \leq C$, then $\left\|u_{1}-u_{2}\right\|_{\left.H^{1}(\Omega)\right)^{2}} \leq K_{2} C_{1}\left\|u_{2}-u_{1}\right\|_{\left(H^{1}(\Omega)\right)^{2}}$ If $u_{1} \neq u_{2}$ we have $K_{2} C_{1} \geq 1$, we can choose $C>0$ very small, such that $K_{2} C_{1}<1$ that is absurd, then the problem $(P)$ has one and only one solution in $\left(W^{1,4}(\Omega)\right)^{2}$.

For conclude the theorem 2.5. We prove that the mathematically solution of $(\mathrm{P})$ is physically accepted. Let $\lambda$ is a eigenvalues of $\nabla u$ we have $|\lambda| \leq|\nabla u|$, if we have $|\lambda| \geq 1$, then

$K C \geq K|\epsilon(u)|_{0, \Omega}^{2} \geq \int_{\Omega}|\nabla u|^{2} \geq \int_{\Omega}|\lambda|^{2} \geq \int_{\Omega} 1=\operatorname{mes}(\Omega)$

Since, $C>0$ is small, then the assumption $|\lambda| \geq 1$ is not satisfied, we conclude that $\operatorname{det}(I+\nabla u)>0$ and afterware [4, chapter.2,page.94] the solution of the problem (P) is physically accepted. 
Acknowledgements. The author is grateful to the anonymous referees for their helpful comments and suggestions

\section{References}

[1] J.M.Ball, Convexity conditions and existence theorems in nonlinear elasticity, Arch.Rational Mech.Anal. 63 $(1976 / 77), 337-403$.

[2] P.G.Ciarlet, Mathematical elasticity. Vol.I.Three dimensional elasticity, Studies in Mathematics and its Applications, 20,North-Holland, Amsterdam, 1988.

[3] P.G.Ciarlet and G.Geymonat, Sur les lois de comportement en élasticité non linéaire compressible, C.R.Acad. Sci. Paris, Sér.II 295(1982), 423-426.

[4] P.G.Ciarlet. Lecture on three-dimensional elasticity-Bombay-1983.

[5] J.E. Marsden and T.J.R. Hughes, Mathematical Foundations of Elasticity, Prentice-Hall, Engelewood Cliffs, 1983.

[6] P.G.Ciarlet and P. Rabier. Les Equations de Von kármán. Lecture Notes in Mathematics; Vol 826. -Berlin, Heidelberg, New York Springer. 1980.

[7] M. Raissouli, Sur un problème mixte de Saint-Venant en élasticité non linéaire multidimensionnelle, Thèse de doctorat, Université Paul Sabatier, Toulouse, 1986.

[8] J.L.Lions and E. Magenes, Problèmes aux limites non homogènes et applications, Travaux et Recherches Mathématiques, No. 18, Vol. 2, Dunod, Paris, 1968.

[9] M.Raissouli and J.Oudaani, On the Saint-Venant problem in nonlinear elasticity with Dirichlet and Neuman boundary conditions. International Journal of Mathematics ,Game Theory and Algebra. Volume 12,Number 4, pp.325-338. 